\title{
Robotic surgery. A reality among us
}

\section{A cirurgia robótica. Uma realidade entre nós}

\author{
Delta Madureira Filho-TCBC/RJ1
}

In 1979, at the newly opened Hospital Universitário da Universidade Federal do Rio de Janeiro (HU-UFRJ), Professor Ugo Pinheiro Guimarães, now retired, was invited by Professor Levão Bogossian for a lecture at the Department of Surgery. During his speech, he uttered these words: "As a boy I was taken by my father to Derby Club (now the region where the Maracanã is) to watch the first flight of the 14-Bis. Santos Dumont flew 500 meters just above the ground and was the big event of that time. I feel privileged because, in this very life, I saw man reach the moon.". In this editorial I want to parody Professor Ugo. In 1990, I attended and participated in the beginning of laparoscopic surgery in Brazil. I feel privileged because, in this very life, only a few years later, I attended another major event in modern surgery, the emergence of Robotic Surgery.

The first time I saw a surgical robot was in the exhibit sector at the Congress of the American College of Surgeons (ACS) in 1998. There was the Da Vinci's console and, at his side, the robot arms on a mannequin. I was excited and I was sure that one day I could use one in Brazil. Back then it was available in only a few medical centers in the US and Europe. In the following Conferences of the ACS and the Society of American Gastrointestinal Endoscopic Surgeons (SAGES), I attended all meetings where the subject robotic surgery was approached. A dream was born!

In 2006, a company that sells medical equipment brought to Rio de Janeiro the Zeus robot and the robotic arm Aesop, models that competing with Da Vinci. Immediately we made contact with this company and we held an event at the Hospital Universitário Clementino Fraga Filho (HUCFF) in association with the Sociedade de Cirurgia Vídeo Endoscópica do Rio de Janeiro (SOCIVERJ), addressing the Theoretical Foundations of Robotic Surgery and performing some procedures on animals (pigs). The Aesop consisted of a robotic arm that could be used alone or coupled to Zeus. When used without Zeus it served to make the camera move under voice command. We got permission to perform the operation in some patients. Our team used it at HUCFF and by Dr. Ricardo Zorron team used it at Hospital Lourenço Jorge. The seed of enthusiasm among young surgeons about robotic surgery was being planted. The Zeus and Asoep were purchased by Intuitive, the company that makes Da Vinci, and removed from circulation. Today, Da Vinci is the only surgical robot in the market.

In 2008, during a meeting of the Academia Nacional de Medicina, Brazil, and the Academia Nacional de Medicina de Portugal, Lisbon, to celebrate the 200th anniversary of D. João IV in Brazil, we had a meeting with the then Secretary of Science and Technology of the State of Rio de Janeiro, who attended the event, and we sensitized him to help us achieve the purchase of Da Vinci to Rio de Janeiro. We carried out a project at UFRJ for the implementation of a multidisciplinary Center for Research in Surgical Robotics, with the participation of doctors, biomedical engineers and technicians from Núcleo de Computação Eletrônica da UFRJ (NCE). The purpose would be the formation of knowledge in the area, involving the School of Medicine, COPPE and NCE. Several meetings were held, but unfortunately it was not possible, for various reasons, to acquire the Da Vinci at that time.

The big dream began to be held in Rio de Janeiro when the Hospital do Instituto Nacional do Câncer (INCA) began negotiating the purchase of the Da Vinci robot. At the time, at a conference held at the Hotel Windsor in 2011, several topics were presented on robotic surgery and Da Vinci simulator (Mimic) was exhibited. The following week, it was possible to organize a one-day event at the Hospital Samaritano in Rio de Janeiro, with theoretical issues about surgical robotics and training in the Mimic simulator. In 2012, the Hospital Samaritano bought the Da Vinci, forming groups of General Surgery, Urology, Gynecology and Head and Neck Surgery. It provided a long and responsible training period for these doctors, bringing to Brazil foreign surgeons to guide their first surgeries. Today, they have held more than 700 robotic surgeries successfully. Recently, the Marcilio Dias Navy Hospital acquired the Da Vinci, and Rio de Janeiro has three hospitals performing robotic surgery.

Robotic surgery is a minimally-invasive procedure that follows the same line of laparoscopic surgery. Long working instruments within trocars introduced through the abdominal wall and / or chest are used. The difference is that in laparoscopy the clamps are manipulated by the surgeon's hand. In robotics, the surgeon controls the robot arms from a distance, sitting at a console with his thumb,

1. Professor Titular de Cirurgia Geral da Escola Médica de Pós Graduação da PUC-Rio, Rio de Janeiro, Brasil. 
index and middle fingers introduced in a device that drives and directs the movements of the robot (assisted robotic surgery).

Early researches on robotic surgery were held in military institutions. It was thought that the main benefit would be to conduct operations at places distant from the patient, for example, in field hospitals during armed conflict or in space stations. Despite the performance of a transatlantic operation, with the surgeon in New York (professor Jacques Marescoux) and the patient in Strasbourg, France, - "Lindbergh Surgery", the name of the first man to cross the Atlantic by plane -, this operation was not repeated due to high cost, the communication being made through optical fiber across the Atlantic Ocean.

The real advantage of robotic surgery is its accuracy. Today it is performed with the surgeon at the console in the same operating in which the patient and the robot arms are. The surgeon's vision is three-dimensional, very good, and superior to laparoscopy. The instruments are accurate and perform movements similar to the human hand (Endowrist). There is no tremor or fatigue. These facts enable advantage over laparoscopic surgery, it being therefore suitable for carrying out complex and difficult operations of being performed by laparoscopy. For the surgeon there is great advantage in ergonomics. He works sitting, relaxed, with his forehead resting on the console display. If he turns his head away from the console, the robot arms stop immediately, allowing the doctor a little break to look at an exam, discuss the case with a colleague, or even make a small snack.

The cost of each operation is still high. One company holds the patent and manufactures the Da Vinci. But like everything else in technology, over time the cost should decrease. I believe that before long the surgical robot will be available in most good hospitals. Most surgeons will have access to its use and simple procedures will also be performed by robotics.

Today, August 2015, in Brazil, operations are performed by robots only in in Sao Paulo, Rio de Janeiro, Porto Alegre, Barretos and, soon, in Fortaleza. I am sure that if one reads this editorial in ten years one will see a major change in the period, ie robotic surgery present in most Brazilian cities, with many different types of surgical robots, specific robots for specific types of operations and surgeons using them routinely, as they do today in laparoscopic surgery. 